\title{
Foliated Quantum Field Theory of Fracton Order
}

\author{
Kevin Slagle $\odot$ \\ Department of Physics and Institute for Quantum Information and Matter, California Institute of Technology, \\ Pasadena, California 91125, USA \\ Walter Burke Institute for Theoretical Physics, California Institute of Technology, Pasadena, California 91125, USA
}

(Received 14 August 2020; accepted 4 February 2021; published 12 March 2021)

\begin{abstract}
We introduce a new kind of foliated quantum field theory (FQFT) of gapped fracton orders in the continuum. FQFT is defined on a manifold with a layered structure given by one or more foliations, which each decompose spacetime into a stack of layers. FQFT involves a new kind of gauge field, a foliated gauge field, which behaves similar to a collection of independent gauge fields on this stack of layers. Gauge invariant operators (and their analogous particle mobilities) are constrained to the intersection of one or more layers from different foliations. The level coefficients are quantized and exhibit a duality that spatially transforms the coefficients. This duality occurs because the FQFT is a foliated fracton order. That is, the duality can decouple $2+1 \mathrm{D}$ gauge theories from the FQFT through a process we dub exfoliation.
\end{abstract}

DOI: 10.1103/PhysRevLett.126.101603

Fracton topological order [1-6] is a phase of matter that exhibits particles with mobility constraints. Such particles include fractons, lineons, and planons, which are energetically constrained to zero-dimensional, one-dimensional, and two-dimensional spatial submanifolds when isolated from other excitations. Fracton research has been motivated as a means for more robust quantum information storage $[5,7,8]$, novel dynamics [9-18], toy models for holography $[19,20]$, exotic materials and fluids [21-33], and connections to quantum gravity [34].

In this work, we focus on gapped [35] type-I [4] fracton models that do not have any gauge-invariant fractal operators [44]. The mobility constraints [47] and other important properties $[48,49]$ of these models have a fundamental dependence on a layering structure of spacetime, known as a foliation structure [50], see Fig. 1. References [51-54] have shown that these fracton phases can be thought of as a topological quantum field theory (TQFT) that is embedded with stacks of interfaces (also called defects) upon which certain anyons are condensed. These interfaces are the so-called leaves (i.e., layers) of the foliation. Therefore, instead of coupling to a metric $g_{\mu \nu}$, these fracton phases are coupled to one or more foliations. For example, the $X$-cube model [4] on a simple cubic lattice is coupled to three flat foliations, but more generic foliations are also allowed [50,55]. This is in contrast to

Published by the American Physical Society under the terms of the Creative Commons Attribution 4.0 International license. Further distribution of this work must maintain attribution to the author(s) and the published article's title, journal citation, and DOI. Funded by SCOAP.
TQFT (without interfaces or defects), which does not couple to a metric or foliation.

Previous works have uncovered field theories for the $X$ cube and other gapped fracton models [54,56-61]. In Ref. [54], the $X$-cube fracton model was generalized to manifolds with arbitrary curved foliations, but formally quantizing the field theory was left as an open problem. Reference [57] later showed how to formally treat the $X$-cube field theory from Ref. [56] as a quantum field theory (QFT) with quantized coefficients.

In this work, we wish to quantize the foliated field theory from Ref. [54]. This task is nontrivial and requires new ideas, such as the introduction of a new kind of foliated gauge field, which behaves like a stack of ordinary gauge fields. We call a QFT with foliated gauge fields a foliated quantum field theory (FQFT).

We also show that the FQFT is a foliated fracton order $[48,50,62,71]$. Foliated fracton orders have ground states for which a local unitary transformation can decouple 2D topological orders from the ground state. In the FQFT, this transformation exhibits an IR duality that decouples
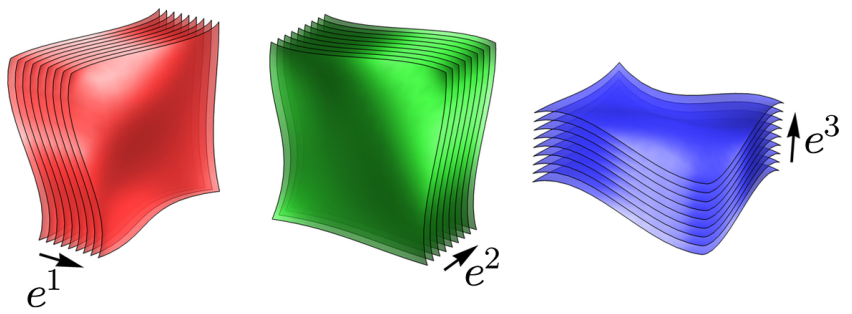

FIG. 1. A depiction of some leaves (colored surfaces) for three different foliations. A foliation consists of an infinite number of infinitesimally spaced layers, which are called leaves. 
$2+1 \mathrm{D}$ gauge theories from the FQFT by giving a coupling constant a piecewise spatial dependence which can be manipulated by the duality.

Foliation field.-A foliation is a decomposition of a manifold into an infinite number of disjoint lowerdimensional submanifolds called leaves. A common example is to decompose $3+1 \mathrm{D}$ spacetime into 3D spatial slices; in this example, the codimension-one leaves can be indexed by the time coordinate.

We will describe a codimension-one foliation using a 1 -form foliation field $e_{\mu}$. The foliation field is analogous to a metric $g_{\mu \nu}$, except $e_{\mu}$ describes a foliation geometry instead of a Riemannian geometry. The leaves of the foliation are defined to be the codimension-one submanifolds that are orthogonal to the foliation field. That is, the tangent vectors $v^{\mu}$ of the leaves are in the null space of the foliation field covector: $v^{\mu} e_{\mu}=0$. The foliation field must never be zero and it must satisfy the following constraint[72]:

$$
e \wedge d e=0
$$

(which can be viewed as a special case of the Frobenius theorem [73]).

More intuition can be obtained by noting that the foliation is invariant under a "gauge transformation" that rescales the foliation field (since this does not affect orthogonality to the leaves):

$$
e \rightarrow \gamma e
$$

where $\gamma$ is a scalar function. It is always possible to apply the above transformation such that within an open ball of spacetime, the foliation fields are closed $(d e=0)$ and can be written as the derivative of a scalar function $f: e=d f$. Locally, $f$ can be thought of as a coordinate that indexes the leaves of the foliation, similar to how a time coordinate indexes time slices of spacetime.

To foliate a torus, the foliation field can be chosen to be closed (e.g., $e=d x$ so that $d e=0$ ). For more exotic foliations, the exterior derivative takes the form $d e=e \wedge \beta$ for some 1 -form $\beta$. The cohomology class of $\beta \wedge d \beta$ is the so-called Godbillon-Vey invariant of the foliation [74,75], which classifies the obstruction to a closed foliation field. Under $e \rightarrow \gamma e, \beta$ transforms as $\beta \rightarrow \beta-d \gamma$.

Multiple simultaneous foliations $e^{k}$ are indexed by the superscript $k=1,2, \ldots, n_{f}$ (Fig. 1). Each foliation satisfies Eq. (1) independently: $e^{k} \wedge d e^{k}=0$. We never implicitly sum over repeated foliation indices $k$.

Foliated QFT.-The foliated QFT (FQFT) Lagrangian is [76]

$$
L=\sum_{k=1}^{n_{f}} \frac{M_{k}}{2 \pi}\left(d B^{k}+n_{k} b\right) \wedge A^{k}+\frac{N}{2 \pi} b \wedge d a
$$

$$
A^{k} \wedge e^{k}=0
$$

$B^{k}$ and $a$ are 1-form gauge fields. (Note that $B^{k}$ is not a magnetic field in this notation; $B^{k}$ has no dependence on $A^{k}$.) $b$ is a 2 -form gauge field. $A^{k}$ are foliated $(1+1)$-form gauge fields, which are locally 2 -forms that obey the constraint Eq. (4). We will show that the physics is equivalent under $n_{k} \sim n_{k}+N$ and that $M_{k}, n_{k}, N \in \mathbb{Z}$ are quantized level coefficients with $m_{k} \equiv\left(n_{k} M_{k} / N\right) \in \mathbb{Z}$ (and $M_{k} \neq 0$ and $N \neq 0$ ). $\sum_{k=1}^{n_{f}}$ sums over the different foliations. Unlike the dynamical gauge fields $\left(A^{k}, B^{k}, a, b\right)$, the foliation field $e_{\mu}$ is nondynamical and is not integrated over in the partition function (analogous to a static metric $g_{\mu \nu}$ ). Similar to a TQFT, FQFT does not couple to a metric.

If $n_{k}=0$, the second term in $L$ describes a $3+1 \mathrm{D} \mathrm{BF}$ theory (which is a field theory for $Z_{N}$ gauge theory or 3D toric code [56,77]), while the first term is a FQFT for a stack of infinitesimally spaced $2+1 \mathrm{D}$ BF theories for each foliation (i.e., a field theory for stacks of $Z_{M_{k}}$ toric codes [78]). When $M_{k}=N$ and $n_{k}=1$, the leaves are coupled to the $3+1 \mathrm{D}$ BF theory, and the resulting theory describes the ground state Hilbert space [79] of the $Z_{N} X$-cube model $[4,54,56,57]$ on any foliation [80] in the limit of infinitesimal lattice spacing. This equivalence can be demonstrated in a number of ways [81] and will be exemplified in this work. Some intuition from coupledlayer constructions of fracton models applies here as well [84-86].

Foliated gauge field: The foliated QFT includes a new kind a gauge field: a foliated $(1+1)$-form gauge field $A^{k}$ for each foliation $k$. A foliated $(1+1)$-form gauge field behaves similarly to a stack of independent 1 -form gauge fields. This is desirable because when $n_{k}=0$, the first term in Eq. (3) should describe a stack of independent 2+1D gauge theories.

Locally, a foliated $(1+1)$-form gauge field $A^{k}$ is a 2-form gauge field that obeys the constraint Eq. (4). Similar to ordinary gauge fields, the exterior derivative $d A^{k}$ is required to be well defined. Note that this requirement does not put any restriction on the continuity of the foliated gauge field $A^{k}$ between leaves of the foliation. For example if $e^{1}=d z$, then the constraint Eq. (4) implies that $A^{1}=\tilde{A}^{1} \wedge d z$ for some 1 -form $\tilde{A}^{1}$, and $\tilde{A}^{1}$ can have arbitrary discontinuities in the $z$ direction since these discontinuities will not contribute to $d A^{1}$ (due to the antisymmetry induced by the wedge product). Furthermore, we allow foliated gauge fields to contain a delta function onto a leaf. For example if $e^{1}=d z$, then $A^{1}=x \delta(z) d y \wedge d z$ is allowed. See Supplemental Material (SM) B [63] for a more formal definition of foliated gauge fields.

Since the first term in Eq. (3) should describe a stack of $2+1 \mathrm{D} \mathrm{BF}$ theories for each $k$ with $n_{k}=0$, the gauge fields $A^{k}$ and $B^{k}$ should effectively have three components 
(since the 1-form gauge fields in 2+1D BF theory have three components). Considering again the example $e^{1}=d z$, we indeed see that the constraint Eq. (4) implies that the foliated $(1+1)$-form has exactly three components: $A^{1}=\left(A_{03}^{1} d t+A_{13}^{1} d x+A_{23}^{1} d y\right) \wedge d z$. The 1-form gauge field $B^{k}$ has four components $\left(B^{1}=B_{0}^{1} d t+B_{1}^{1} d x+B_{2}^{1} d y+B_{3}^{1} d z\right)$. However there is a gauge symmetry $B^{k} \rightarrow \alpha^{k}$ for an arbitrary foliated $(0+1)$-form $\alpha^{k}$, which locally satisfies $\alpha^{k} \wedge e^{k}=0$ (i.e., locally $\alpha^{k}=\tilde{\alpha}^{k} e^{k}$ for some scalar $\tilde{\alpha}^{k}$ ). This makes the $d z$ component an unimportant gauge redundancy. Therefore, $A^{k}$ and $B^{k}$ both effectively have three components (for each foliation $k$ ), as desired.

Fractons and gauge invariant operators: The set of gauge symmetries determines the set of gauge invariant operators. In ordinary topological QFT (e.g., Chern-Simons theory), gauge invariant operators can be smoothly deformed into any shape. However, in a foliated QFT, the gauge invariant operators are often constrained to the intersection of one or more leaves of different foliations.

Gauge invariant operators can be interpreted as moving topological excitations around in spacetime. Therefore, the rigidity of the gauge invariant operators is analogous to the mobility constraints of the fracton, lineon, and planon particles.

The gauge transformations of the FQFT are

$$
\begin{aligned}
A^{k} & \rightarrow A^{k}+d \zeta^{k} \\
B^{k} & \rightarrow B^{k}+d \chi^{k}-n_{k} \mu+\alpha^{k} \\
a & \rightarrow a+d \lambda-\sum_{k} m_{k} \zeta^{k} \\
b & \rightarrow b+d \mu
\end{aligned}
$$

where $m_{k} \equiv\left(n_{k} M_{k} / N\right) \cdot \chi^{k}$ and $\lambda$ are arbitrary 0-form gauge fields, while $\mu$ is an arbitrary 1-form gauge field. $\zeta^{k}$ and $\alpha^{k}$ are foliated $(0+1)$-form gauge fields. Locally, $\zeta^{k}$ are 1-form gauge fields that satisfy the constraint[87] $\zeta^{k} \wedge e^{k}=0$, and similar for $\alpha^{k}$.

Consider the following string operator:

$$
W=e^{i q \oint_{\mathcal{M}_{1}^{F}} a}
$$

where $\mathcal{M}_{1}^{F}$ is a one-dimensional manifold described below. Large gauge transformations imply that the charge $q$ is an integer. A nonlocal "equation of motion" (from integrating out $b$ ) shows that $W=1$ when $q$ is an integer multiple of $N$ [88]. Therefore $W$ only depends on $q$ modulo $N$. After a gauge transformation, $W \rightarrow W \exp \left[i \oint_{\mathcal{M}_{1}^{F}}\left(d \lambda-\sum_{k} m_{k} \zeta^{k}\right)\right]$. The first term, $\oint_{\mathcal{M}_{1}^{F}} d \lambda$, is invariant if $\mathcal{M}_{1}^{F}$ is a closed loop. The second term, $\oint_{\mathcal{M}_{1}^{F}} \sum_{k} m_{k} \zeta^{k}$, is invariant if the tangent vectors $v^{\mu}$ of $\mathcal{M}_{1}^{F}$ are in the null space of each $m_{k} \xi^{k}$, i.e., $v^{\mu} m_{k} \zeta_{\mu}^{k}=0$. But locally, $\zeta_{\mu}^{k}=\tilde{\zeta}^{k} e_{\mu}^{k}$ for some scalar $\tilde{\zeta}^{k}$. Therefore the second term is gauge invariant if for each $k$ with $m_{k} \neq 0$, the loop $\mathcal{M}_{1}^{F}$ is supported on a single leaf of the $k$ th foliation [since then $v^{\mu} m_{k} \zeta_{\mu}^{k} \propto v^{\mu} e_{\mu}^{k}$ and $v^{\mu} e_{\mu}^{k}=0$ by the definition of $e^{k}$ above Eq. (1)].

Therefore, if there are $n$ foliations with $m_{k} \neq 0$, then the string operator [Eq. (6)] and the particle it transports are bound to the intersection of $n$ leaves. If there are three or more spatial [89] foliations (that are spatially transverse [90]) as in Fig. 1, then this string operator can move fractons in time (assuming time is periodic), but it cannot move fractons spatially. When $n_{k}=1$ and $M_{k}=N$, this fracton is equivalent to the $X$-cube fracton [4] for any foliation [80]. It has been proven that all compact orientable 3-manifolds admit a total foliation (i.e., three transverse foliations) [91], which implies that all such manifolds admit an FQFT with fractons.

Consider a different string operator:

$$
T=e^{i \oint_{\mathcal{M}_{1}^{L}} \sum_{k} q_{k} B^{k}} .
$$

Large gauge transformations imply that the charges $q_{k} \in \mathbb{Z}$ are integers. The $B^{k} \rightarrow B^{k}+d \chi^{k}$ gauge transformation shows that $\mathcal{M}_{1}^{L}$ must be a closed loop. The $B^{k} \rightarrow B^{k}+$ $\alpha^{k}$ gauge transformation [where $\alpha^{k}=\tilde{\alpha}^{k} e^{k}$ (locally) is a foliated $(0+1)$-form] shows that $\mathcal{M}_{1}^{L}$ is supported on the intersection of $n$ leaves, where $n$ is the number of foliations $k$ with nonzero $q_{k} \neq 0$. Finally, the $B^{k} \rightarrow B^{k}-n_{k} \mu$ gauge transformation implies that $\sum_{k} q_{k} n_{k}=0$. Therefore, the set of allowed charge vectors forms an Abelian group $G=\left\{q \in \mathbb{Z}^{n_{f}} \mid \sum_{k} q_{k} n_{k}=0\right\}$.

A nonlocal "equation of motion" (from integrating out $A^{k}$ ) shows [88] that $T=1$ when $q_{k} \in M_{k} \mathbb{Z}$ (and $\sum_{k} q_{k} n_{k}=0$ ). Thus, the trivial charge vectors form a subgroup $N=\left\{q \in G \mid q_{k} \in M_{k} \mathbb{Z}\right\} \triangleleft G$. Since both of these groups $\left(G\right.$ and $N$ ) are isomorphic to $\mathbb{Z}^{n_{f}-1}$ (or $\mathbb{Z}^{n_{f}}$ if $n_{k}=0$ ), their quotient group $G / N$ of physically distinct charge vectors is a finite Abelian group (i.e., $G / N$ is isomorphic to $Z_{r_{1}} \times \cdots \times Z_{r_{n_{f}-1}}$ for some integers $r_{i} \in \mathbb{N}$ ).

In the $Z_{N} X$-cube model example with three foliations and $n_{k}=1$ and $M_{k}=N$, the allowed charge vectors are

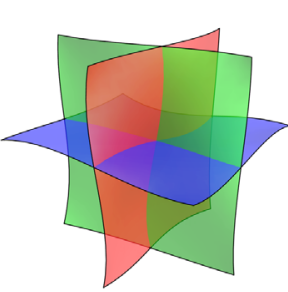

(a)

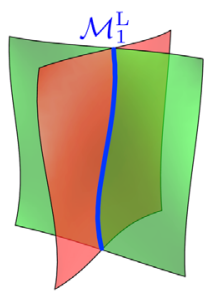

(b)

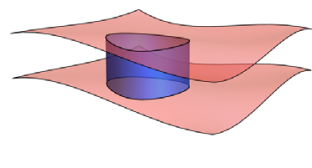

(c)
FIG. 2. Spatial pictures of: (a) Three leaves intersecting at a point. (b) A one-dimensional manifold $\mathcal{M}_{1}^{L}$ (blue) at the intersection of two leaves (red and green). (c) A two-dimensional manifold $\mathcal{M}_{2}^{P}$ (blue) with boundaries supported on leaves (red). 
spanned by $q_{k}^{(X)}=(0,1,-1)$ and $q_{k}^{(Z)}=(1,-1,0)$. These particles are bound to a pair of leaves [Fig. 2(b)] and are therefore restricted to spatially only move along 1D lines (for spatial foliations). These are $Z_{N} X$-cube lineons. For the standard three flat foliations $\left(e^{1}=d x, e^{2}=d y\right.$, $\left.e^{3}=d z\right), q_{k}^{(X)}$ and $q_{k}^{(Z)}$ can move only in the $X$ and $Z$ directions, respectively; and their sum $q_{k}^{(X)}+q_{k}^{(Z)}=$ $(1,0,-1)$ can only move in the $Z$ direction. This is analogous to the $X$-cube model where the composition of an $X$-axis lineon with a $Z$-axis lineon is a $Y$-axis lineon. The physics generalizes naturally to $n$ foliations: the charge vectors are spanned by $n-1$ vectors of the form $(\cdots, 0,1,-1,0, \ldots)$. Note that even if a charge vector has three nonzero components, it is not a fracton; instead, it is the composition of at most two lineons.

Even for an arbitrary number of foliations and coefficients $n_{k}$, it is always possible to decompose a charge vector $q_{k}$ into lineon and planon charges (which have at most two nonzero elements $q_{k} \neq 0$ ). See SM E [63] for a proof. Therefore, the string operator $T$ only describes lineons (or composites of lineons and planons), but never fractons.

Other gauge invariant operators include

$$
T^{\prime}=e^{i \oint_{\mathcal{M}_{2}} b} \quad W^{\prime}=e^{i \oint_{\mathcal{M}_{2}^{P}} A^{k}} .
$$

$\oint_{\mathcal{M}_{2}} b$ denotes an integral of $b$ over a closed 2-manifold $\mathcal{M}_{2} . \oint_{\mathcal{M}_{2}^{p}} A^{k}$ denotes an integral of $A^{k}$ over a 2-manifold $\mathcal{M}_{2}^{P}$ with boundaries that must each be supported on a single leaf of the foliation $k$, as in Fig. 2(c). $T^{\prime}$ wraps a string excitation around $\mathcal{M}_{2}$. In the $X$-cube model example, $T^{\prime}$ measures the number of fractons inside $\mathcal{M}_{2}$. In the $X$-cube lattice model, this operator is a complicated operator that wraps a loop of many lineon excitations around $\mathcal{M}_{2}$ [92]. In the $X$-cube example, $W^{\prime}$ moves a pair of $X$-cube fractons [93] around the top and bottom boundaries of the blue 2-manifold $\mathcal{M}_{2}^{P}$ shown in Fig. 2(c).

See SM C [63] for more general operators and a different approach to understanding the particle mobility constraints.

Level quantization: Now we study the quantization of the level coefficients $M_{k}, n_{k}$, and $N$. First note that $m_{k} \equiv n_{k} M_{K} / N$ and $n_{k}$ appear as coefficients in the gauge transformations [Eq. (5)] of compact gauge fields $\left(a\right.$ and $B^{k}$ ). This implies that $m_{k}, n_{k} \in \mathbb{Z}$.

The Lagrangian transforms under the gauge transformations as

$$
\begin{aligned}
L \rightarrow L^{\prime}= & L+\sum_{k} \frac{M_{k}}{2 \pi}\left(d B^{k} \wedge d \zeta^{k}+d \chi^{k} \wedge d A^{k}\right) \\
& +\frac{N}{2 \pi}(d b \wedge d \lambda+d \mu \wedge d a) .
\end{aligned}
$$

Locally, the new terms are total derivatives. But since these are derivatives of gauge fields, their integral over a closed manifold can be nonzero. However, the integral is quantized such that the change in the action is an integer multiple of $2 \pi M_{k}$ plus an integer multiple of $2 \pi N$. Therefore, the partition function $Z=e^{i \int L}$ is gauge invariant if $M_{k}, N \in \mathbb{Z}$.

The equations of motion that result from integrating out $a$ and $B^{k}$ imply [88] that locally $d b=d A^{k}=0$ and globally the operators in Eq. (8) are quantized:

$$
\oint_{\mathcal{M}_{2}} b \in \frac{2 \pi}{N} \mathbb{Z} \quad \oint_{\mathcal{M}_{2}^{P}} A^{k} \in \frac{2 \pi}{M_{k}} \mathbb{Z} .
$$

Together, these local and global equations of motion show that the $b \wedge A^{k}$ term in the FQFT action [Eq. (3)] is quantized as follows:

$$
\frac{M_{k} n_{k}}{2 \pi} \int b \wedge A^{k} \in 2 \pi \frac{n_{k}}{N} \mathbb{Z} .
$$

This implies that the action is invariant under the following identification: $n_{k} \sim n_{k}+N$.

Exfoliation.-Reference [50] showed that a finite-depth local unitary transformation can map between the ground states of (1) an $X$-cube model of lattice length $L_{0}$ in one direction, and (2) an $X$-cube model of lattice length $L_{0}-1$ in the same direction along with a decoupled layer of toric code (and some trivial decoupled qubits). We will refer to this process as exfoliation. In high-energy terminology, exfoliation corresponds to an IR duality that decouples $2+1 \mathrm{D}$ gauge theories from a 3D FQFT. A fracton order that admits exfoliation is said to be a foliated fracton order [50,62,71]. The $X$-cube model is a foliated fracton order that is foliated by toric code layers [50,94].

We now show that the FQFT is a foliated fracton order by exfoliating $2+1 \mathrm{D}$ BF theories. For simplicity, consider a flat foliation $e^{1}=d z$ (which may coexist with other foliations $e^{k}$ ). We want to demonstrate a duality from a FQFT with constant $n_{1} \in \mathbb{Z}$ to a FQFT with a spatially dependent $\tilde{n}_{1}(z)$ that is zero within $z_{1}<z<z_{2}$ :

$$
n_{1} \leftrightarrow \tilde{n}_{1}(z)= \begin{cases}n_{1} & z \leq z_{1} \quad \text { or } \quad z \geq z_{2} \\ 0 & z_{1}<z<z_{2} .\end{cases}
$$

On the right-hand side of the duality, the $A^{1}$ and $B^{1}$ fields within $z_{1}<z<z_{2}$ are decoupled from the rest of the fields. The equations of motion for $A^{1}$ and $B^{1}$ are $d A^{1}=$ $d B^{1} \wedge e^{1}=0$ within $z_{1}<z<z_{2}$. These equations of motion do not contain $z$ derivatives $\partial_{z}$ [recall $A^{1} \wedge e^{1}=$ 0 from Eq. (4)], which shows that $A^{1}$ and $B^{1}$ at different $z$ are completely decoupled. These decoupled fields constitute an exfoliated stack of infinitesimally spaced $2+1 \mathrm{D}$ $\mathrm{BF}$ theories. 
The duality results from the following transformation:

$$
\begin{aligned}
& a \leftrightarrow \tilde{a}= \begin{cases}a & z \leq z_{1} \quad \text { or } \quad z_{2} \leq z \\
a+m_{1} \int_{z_{1}}^{z} A^{1} & z_{1}<z<z_{2}\end{cases} \\
& A^{1} \leftrightarrow \tilde{A}^{1}=A^{1}+\delta\left(z-z_{2}\right) \int_{z_{1}}^{z_{2}} d z A^{1} \\
& B^{1} \leftrightarrow \tilde{B}^{1}= \begin{cases}B^{1} & z \leq z_{1} \quad \text { or } z_{2} \leq z \\
B^{1}-B^{1}\left(z_{2}\right)+n_{1} \int_{z}^{z_{2}} b & z_{1}<z<z_{2} .\end{cases}
\end{aligned}
$$

We are using a notation where the integrals above are defined as $\left(\int_{z_{1}}^{z} A^{1}\right)_{\mu} \equiv \int_{z_{1}}^{z} A_{3 \mu}^{1} d z,\left(\int_{z_{1}}^{z_{2}} d z A^{1}\right)_{\mu \nu}=$ $\int_{z_{1}}^{z_{2}} d z A_{\mu \nu}^{1}$, and $\left(\int_{z}^{z_{2}} b\right)_{\mu} \equiv \int_{z}^{z_{2}} b_{3 \mu} d z$ [97]. In order for this definition to make sense, we have implicitly chosen a flat connection to parallel transport the gauge fields. $B^{1}\left(z_{2}\right)$ is shorthand for $B^{1}\left(t, x, y, z_{2}\right)$, just as $B^{1}$ is shorthand for $B^{1}(t, x, y, z)$. In SM G.1 [63], we show that the above equation transforms the equations of motion according to Eq. (12), which demonstrates the exfoliation duality.

Note that since the duality acts nonlocally on the fields, the locality of some gauge invariant operators can change. Indeed, this must occur because $\tilde{n}_{1}=0$ will result in less rigidity constraints on the gauge invariant operators. See SM G [63] for examples and further discussion.

Conclusion.-We have introduced a generic foliated QFT (FQFT) that is capable of describing a large class of foliated gapped fracton models on foliated manifolds. We also demonstrated a novel duality that spatially transforms the level coefficients, which shows that the FQFT is a foliated fracton order [50,62,71].

Many future directions remain. Additional terms can be added to the FQFT Lagrangian to realize more exotic fracton models [71,98-103]. The fractonic Higgs mechanism [104,105] could be revisited now that we understand gapped fracton orders on curved foliations $[50,51,54,106]$ and $U(1)$ fracton models [36-42] on curved space [107]. Finally, FQFT could provide further insight on other works, such as the study of boundaries of fracton models [108] or models in higher dimensions [109].

We thank Shu-Heng Shao, Nathan Seiberg, Ho Tat Lam, Pranay Gorantla, Po-Shen Hsin, Anton Kapustin, Xie Chen, and Wilbur Shirley for helpful discussion. K. S. is supported by the Walter Burke Institute for Theoretical Physics at Caltech.

[1] R. M. Nandkishore and M. Hermele, Fractons, Annu. Rev. Condens. Matter Phys. 10, 295 (2019).

[2] M. Pretko, X. Chen, and Y. You, Fracton phases of matter, Int. J. Mod. Phys. A 35, 2030003 (2020).

[3] S. Vijay, J. Haah, and L. Fu, A new kind of topological quantum order: A dimensional hierarchy of quasiparticles built from stationary excitations, Phys. Rev. B 92, 235136 (2015).

[4] S. Vijay, J. Haah, and L. Fu, Fracton topological order, generalized lattice gauge theory, and duality, Phys. Rev. B 94, 235157 (2016).

[5] J. Haah, Local stabilizer codes in three dimensions without string logical operators, Phys. Rev. A 83, 042330 (2011).

[6] S. Bravyi, B. Leemhuis, and B. M. Terhal, Topological order in an exactly solvable 3D spin model, Ann. Phys. (Amsterdam) 326, 839 (2011).

[7] S. Bravyi and J. Haah, Quantum Self-Correction in the 3d Cubic Code Model, Phys. Rev. Lett. 111, 200501 (2013).

[8] B. J. Brown, D. Loss, J. K. Pachos, C. N. Self, and J. R. Wootton, Quantum memories at finite temperature, Rev. Mod. Phys. 88, 045005 (2016).

[9] C. Chamon, Quantum Glassiness in Strongly Correlated Clean Systems: An Example of Topological Overprotection, Phys. Rev. Lett. 94, 040402 (2005).

[10] A. Prem, J. Haah, and R. Nandkishore, Glassy quantum dynamics in translation invariant fracton models, Phys. Rev. B 95, 155133 (2017).

[11] S. Pai, M. Pretko, and R. M. Nandkishore, Localization in Fractonic Random Circuits, Phys. Rev. X 9, 021003 (2019).

[12] A. Gromov, A. Lucas, and R. M. Nandkishore, Fracton hydrodynamics, Phys. Rev. Research 2, 033124 (2020).

[13] S. Pai and M. Pretko, Dynamical Scar States in Driven Fracton Systems, Phys. Rev. Lett. 123, 136401 (2019).

[14] H. He, Y. You, and A. Prem, Lieb-Schultz-Mattis-type constraints on fractonic matter, Phys. Rev. B 101, 165145 (2020).

[15] O. Dubinkin, J. May-Mann, and T. L. Hughes, Lieb Schultz Mattis-type theorems and other non-perturbative results for strongly correlated systems with conserved dipole moments, arXiv:2001.04477.

[16] H. Shackleton and M. S. Scheurer, Protection of paritytime symmetry in topological many-body systems: NonHermitian toric code and fracton models, Phys. Rev. Research 2, 033022 (2020).

[17] J. Feldmeier, P. Sala, G. De Tomasi, F. Pollmann, and M. Knap, Anomalous Diffusion in Dipole- and Higher-MomentConserving Systems, Phys. Rev. Lett. 125, 245303 (2020).

[18] J.-K. Yuan, S. A. Chen, and P. Ye, Fractonic superfluids, Phys. Rev. Research 2, 023267 (2020).

[19] H. Yan, Hyperbolic fracton model, subsystem symmetry, and holography, Phys. Rev. B 99, 155126 (2019).

[20] H. Yan, Geodesic string condensation from symmetric tensor gauge theory: A unifying framework of holographic toy models, Phys. Rev. B 102, 161119(R) (2020).

[21] H. Yan, O. Benton, L. D. C. Jaubert, and N. Shannon, Rank-2 U(1) Spin Liquid on the Breathing Pyrochlore Lattice, Phys. Rev. Lett. 124, 127203 (2020).

[22] M. Pretko and L. Radzihovsky, Fracton-Elasticity Duality, Phys. Rev. Lett. 120, 195301 (2018).

[23] M. Pretko, Z. Zhai, and L. Radzihovsky, Crystal-to-fracton tensor gauge theory dualities, Phys. Rev. B 100, 134113 (2019).

[24] G. B. Halász, T. H. Hsieh, and L. Balents, Fracton Topological Phases from Strongly Coupled Spin Chains, Phys. Rev. Lett. 119, 257202 (2017). 
[25] Y. Fuji, Anisotropic layer construction of anisotropic fracton models, Phys. Rev. B 100, 235115 (2019).

[26] K. Slagle and Y. B. Kim, Fracton topological order from nearest-neighbor two-spin interactions and dualities, Phys. Rev. B 96, 165106 (2017).

[27] A. Prem, S. Vijay, Y.-Z. Chou, M. Pretko, and R. M. Nandkishore, Pinch point singularities of tensor spin liquids, Phys. Rev. B 98, 165140 (2018).

[28] D. Nguyen, A. Gromov, and S. Moroz, Fracton-elasticity duality of two-dimensional superfluid vortex crystals: Defect interactions and quantum melting, SciPost Phys. 9, 076 (2020).

[29] D. Doshi and A. Gromov, Vortices and fractons, arXiv: 2005.03015.

[30] S. Pankov, R. Moessner, and S. L. Sondhi, Resonating singlet valence plaquettes, Phys. Rev. B 76, 104436 (2007).

[31] C. $\mathrm{Xu}$ and C. Wu, Resonating plaquette phases in SU(4) Heisenberg antiferromagnet, Phys. Rev. B 77, 134449 (2008).

[32] J. Sous and M. Pretko, Fractons from polarons, Phys. Rev. B 102, 214437 (2020).

[33] Y. You and F. von Oppen, Building fracton phases by Majorana manipulation, Phys. Rev. Research 1, 013011 (2019).

[34] M. Pretko, Emergent gravity of fractons: Mach's principle revisited, Phys. Rev. D 96, 024051 (2017).

[35] We will not study the gapless $U(1)$ fracton models [36-43], which are analogous to $U(1)$ Maxwell gauge theory. Gapped fracton models are analogous to $Z_{N}$ gauge theory, BF theory, and Chern-Simons theory.

[36] M. Pretko, Subdimensional particle structure of higher rank U(1) spin liquids, Phys. Rev. B 95, 115139 (2017).

[37] A. Rasmussen, Y.-Z. You, and C. Xu, Stable gapless Bose liquid phases without any symmetry, arXiv:1601.08235.

[38] M. Pretko, Generalized electromagnetism of subdimensional particles: A spin liquid story, Phys. Rev. B 96, 035119 (2017).

[39] L. Radzihovsky and M. Hermele, Fractons from Vector Gauge Theory, Phys. Rev. Lett. 124, 050402 (2020).

[40] N. Seiberg and S.-H. Shao, Exotic U(1) symmetries, duality, and fractons in $3+1$-dimensional quantum field theory, SciPost Phys. 9, 46 (2020).

[41] N. Seiberg, Field theories with a vector global symmetry, SciPost Phys. 8, 050 (2020).

[42] M. Pretko, Finite-temperature screening of U(1) fractons, Phys. Rev. B 96, 115102 (2017).

[43] T. Griffin, K. T. Grosvenor, P. Hořava, and Z. Yan, Scalar field theories with polynomial shift symmetries, Commun. Math. Phys. 340, 985 (2015).

[44] See Refs. [4,5,45,46] for gapped fracton models with fractal operators.

[45] B. Yoshida, Exotic topological order in fractal spin liquids, Phys. Rev. B 88, 125122 (2013).

[46] K. T. Tian, E. Samperton, and Z. Wang, Haah codes on general three-manifolds, Ann. Phys. (Amsterdam) 412, 168014 (2020).

[47] W. Shirley, K. Slagle, and X. Chen, Foliated fracton order from gauging subsystem symmetries, SciPost Phys. 6, 041 (2019).
[48] W. Shirley, K. Slagle, and X. Chen, Universal entanglement signatures of foliated fracton phases, SciPost Phys. 6, 15 (2019).

[49] S. Pai and M. Hermele, Fracton fusion and statistics, Phys. Rev. B 100, 195136 (2019).

[50] W. Shirley, K. Slagle, Z. Wang, and X. Chen, Fracton Models on General Three-Dimensional Manifolds, Phys. Rev. X 8, 031051 (2018).

[51] D. Aasen, D. Bulmash, A. Prem, K. Slagle, and D. J. Williamson, Topological defect networks for fractons of all types, Phys. Rev. Research 2, 043165 (2020).

[52] X.-G. Wen, Systematic construction of gapped nonliquid states, Phys. Rev. Research 2, 033300 (2020).

[53] J. Wang, Non-liquid cellular states, arXiv:2002.12932.

[54] K. Slagle, D. Aasen, and D. Williamson, Foliated field theory and string-membrane-net condensation picture of fracton order, SciPost Phys. 6, 043 (2019).

[55] K. Slagle and Y. B. Kim, X-cube model on generic lattices: Fracton phases and geometric order, Phys. Rev. B 97, 165106 (2018).

[56] K. Slagle and Y. B. Kim, Quantum field theory of X-cube fracton topological order and robust degeneracy from geometry, Phys. Rev. B 96, 195139 (2017).

[57] N. Seiberg and S.-H. Shao, Exotic $\mathbb{Z}_{N}$ symmetries, duality, and fractons in $3+1$-dimensional quantum field theory, SciPost Phys. 10, 3 (2021).

[58] P. Gorantla, H. T. Lam, N. Seiberg, and S.-H. Shao, More exotic field theories in $3+1$ dimensions, SciPost Phys. 9, 73 (2020).

[59] W. B. Fontana, P. R. S. Gomes, and C. Chamon, Lattice Clifford fractons and their Chern-Simons-like theory, arXiv:2006.10071.

[60] Y. You, T. Devakul, S. L. Sondhi, and F. J. Burnell, Fractonic Chern-Simons and BF theories, Phys. Rev. Research 2, 023249 (2020).

[61] Y. You, T. Devakul, F. J. Burnell, and S. L. Sondhi, Symmetric fracton matter: Twisted and enriched, Ann. Phys. (Amsterdam) 416, 168140 (2020).

[62] See SM F [63] and Section II of Ref. [48] for more details on foliated fracton phases.

[63] See Supplemental Material at http://link.aps.org/ supplemental/10.1103/PhysRevLett.126.101603 for detailed calculations and extended discussion, which includes Refs. [64-70].

[64] E. Witten, The problem of gauge theory, arXiv:0812.4512.

[65] R. Dijkgraaf and E. Witten, Topological gauge theories and group cohomology, Commun. Math. Phys. 129, 393 (1990).

[66] A. Kapustin and N. Seiberg, Coupling a QFT to a TQFT and duality, J. High Energy Phys. 04 (2014) 001.

[67] C. Cordova, D. S. Freed, H. T. Lam, and N. Seiberg, Anomalies in the space of coupling constants and their dynamical applications I, SciPost Phys. 8, 1 (2020).

[68] G. Vidal, Entanglement Renormalization, Phys. Rev. Lett. 99, 220405 (2007).

[69] M. Aguado and G. Vidal, Entanglement Renormalization and Topological Order, Phys. Rev. Lett. 100, 070404 (2008).

[70] A. Dua, P. Sarkar, D. J. Williamson, and M. Cheng, Bifurcating entanglement-renormalization group flows of 
fracton stabilizer models, Phys. Rev. Research 2, 033021 (2020).

[71] W. Shirley, K. Slagle, and X. Chen, Twisted foliated fracton phases, Phys. Rev. B 102, 115103 (2020).

[72] We use differential form notation throughout this work. In components, Eq. (1) can be written as $\epsilon^{\alpha \beta \gamma \delta} e_{\beta} \partial_{\gamma} e_{\delta}=0$ where $\epsilon$ is the Levi-Civita symbol.

[73] T. Frankel, The Geometry of Physics: An Introduction (Cambridge University Press, Cambridge, England, 2011).

[74] C. Godbillon and J. Vey, Un invariant des feuilletages de codimension 1, C. R. Acad. Sci. Paris 273, 92 (1971).

[75] D. Kotschick, Godbillon-Vey invariants for families of foliations, arXiv:math/0111137.

[76] In components, $L=\left[\sum_{k=1}^{n_{f}}\left(M_{k} / 2 \pi\right)\left(\partial_{\alpha} B_{\beta}^{k}+n_{k} b_{\alpha \beta}\right) A_{\gamma \delta}^{k}+\right.$ $\left.(N / 2 \pi) b_{\alpha \beta} \partial_{\gamma} a_{\delta}\right] \epsilon^{\alpha \beta \gamma \delta} d^{4} x$, and Eq. (4) can be written as $\epsilon^{\alpha \beta \gamma \delta} A_{\beta \gamma}^{k} e_{\delta}^{k}=0$. A lattice model for this FQFT was given in Appendix A of Ref. [54]. The Lagrangian can alternatively be written as $L=\sum_{k}\left(M_{k} / 2 \pi\right) B^{k} \wedge d A^{k}+(N / 2 \pi)$ $b \wedge\left(d a+\sum_{k} m_{k} A^{k}\right)$.

[77] T. Banks and N. Seiberg, Symmetries and strings in field theory and gravity, Phys. Rev. D 83, 084019 (2011).

[78] A. Y. Kitaev, Fault-tolerant quantum computation by anyons, Ann. Phys. (N.Y.) 303, 2 (2003).

[79] There are no excitations in the FQFT; the FQFT Hilbert space only consists of degenerate ground states. The same is true of BF theory (or Chern-Simons theory), which describes the ground state Hilbert space of toric code. However, string operators can be thought of as moving particles around spacetime.

[80] See, e.g., Fig. 3(b) of Ref. [55] for a four-foliated $X$-cube model where $e^{1}=+\frac{1}{2} d x-(\sqrt{3} / 2) d y, \quad e^{2}=-\frac{1}{2} d x-$ $(\sqrt{3} / 2) d y, e^{3}=d y, e^{4}=d z$.

[81] One can show [82] that the FQFT with $M_{k}=N$ and $n_{k}=1$ for three flat foliations is dual to the $X$-cube QFT $[56,57]$. The FQFT is very closely related (see SM H [63]) to the foliated field theory in Ref. [54], in which an explicit connection to a string-membrane-net lattice model was shown in Sec. III. C. 3, and Sec. III. C. 2 proved that the string-membrane-net model has the same ground states (up to generalized local unitary [83]) as the $X$-cube model.

[82] S.-H. Shao, P. Gorantla, H. Tat Lam, and N. Seiberg (private communication).

[83] X. Chen, Z.-C. Gu, and X.-G. Wen, Local unitary transformation, long-range quantum entanglement, wave function renormalization, and topological order, Phys. Rev. B 82, 155138 (2010).

[84] S. Vijay, Isotropic layer construction and phase diagram for fracton topological phases, arXiv:1701.00762.

[85] H. Ma, E. Lake, X. Chen, and M. Hermele, Fracton topological order via coupled layers, Phys. Rev. B 95, 245126 (2017).

[86] A. Prem, S.-J. Huang, H. Song, and M. Hermele, Cage-Net Fracton Models, Phys. Rev. X 9, 021010 (2019).

[87] A more relaxed constraint $d \zeta^{k} \wedge e^{k}=0$ is sufficient to guarantee that $A^{k} \wedge e^{k}=0$ is preserved. However, this relaxed constraint would allow transformations such as $\zeta^{k}=c d z$ for any $c \in \mathbb{R}$ (i.e. $c$ is not quantized) on a manifold with periodic boundaries in $z$. This would be undesirable since it would make Eq. (6) not gauge invariant, which would be inconsistent with the lattice model version of this theory [54].

[88] See SM D1 [63] for details.

[89] A spatial foliation is a foliation that has no time component, i.e., $e^{k}(\hat{t})=e_{\mu}^{k} \hat{t}^{\mu}=0$ where $\hat{t}^{\mu}$ is a vector pointing in the time direction.

[90] By spatially transverse, we mean that the spatial foliation of 2D leaves is transverse. Transverse means that when $n$ leaves intersect at a point, then the intersection of the tangent spaces of the $n$ leaves at this point is just the null vector. [91].

[91] D. Hardorp, All Compact Orientable Three Dimensional Manifolds Admit Total Foliations, Memoirs of the American Mathematical Society, Vol. 26 (American Mathematical Society, Providence, 1980).

[92] Although an isolated lineon can only move along a straight line without creating additional excitations, a string of many lineons can move more freely, especially when additional excitations are allowed to be created.

[93] A pair of $X$-cube fractons can form a planon, which has 2D mobility.

[94] See Refs. [47,71,95,96] for more examples of foliated fracton orders.

[95] T. Wang, W. Shirley, and X. Chen, Foliated fracton order in the Majorana checkerboard model, Phys. Rev. B 100, 085127 (2019).

[96] W. Shirley, K. Slagle, and X. Chen, Foliated fracton order in the checkerboard model, Phys. Rev. B 99, 115123 (2019).

[97] In Eq. (13), we use the convention that integrals do not pick up delta functions on their end points; e.g., $\int_{0}^{1} \delta(x) d x=0$.

[98] D. J. Williamson and M. Cheng, Designer non-Abelian fractons from topological layers, arXiv:2004.07251.

[99] A. Prem and D. Williamson, Gauging permutation symmetries as a route to non-Abelian fractons, SciPost Phys. 7, 068 (2019).

[100] D. Bulmash and M. Barkeshli, Gauging fractons: Immobile non-Abelian quasiparticles, fractals, and position-dependent degeneracies, Phys. Rev. B 100, 155146 (2019).

[101] D. T. Stephen, J. Garre-Rubio, A. Dua, and D. J. Williamson, Subsystem symmetry enriched topological order in three dimensions, Phys. Rev. Research 2, 033331 (2020).

[102] N. Tantivasadakarn and S. Vijay, Searching for fracton orders via symmetry defect condensation, Phys. Rev. B 101, 165143 (2020).

[103] T. Devakul, W. Shirley, and J. Wang, Strong planar subsystem symmetry-protected topological phases and their dual fracton orders, Phys. Rev. Research 2, 012059 (R) (2020).

[104] D. Bulmash and M. Barkeshli, Higgs mechanism in higher-rank symmetric U(1) gauge theories, Phys. Rev. B 97, 235112 (2018).

[105] H. Ma, M. Hermele, and X. Chen, Fracton topological order from the Higgs and partial-confinement mechanisms of rank-two gauge theory, Phys. Rev. B 98, 035111 (2018). 
[106] D. Radicevic, Systematic constructions of fracton theories, arXiv:1910.06336.

[107] K. Slagle, A. Prem, and M. Pretko, Symmetric tensor gauge theories on curved spaces, Ann. Phys. (Amsterdam) 410, 167910 (2019).
[108] D. Bulmash and T. Iadecola, Braiding and gapped boundaries in fracton topological phases, Phys. Rev. B 99, 125132 (2019).

[109] M.-Y. Li and P. Ye, Fracton physics of spatially extended excitations, Phys. Rev. B 101, 245134 (2020). 